\title{
Autopercepção da qualidade de vida de pacientes submetidos à artroplastia total do joelho
}

\author{
Patient self-perception of the quality of life after total knee arthroplasty \\ Autopercepción de la calidad de vida de pacientes luego de una artroplastia total de rodilla
}

\author{
Eli Ávila SOUZA JúNIOR \\ Guilherme Ferreira SIMÕES \\ Maíra Soares TORRES \\ Daniel Soares BAUMFELD \\ Tulio Vinícius de Oliveira CAMPOS \\ Marco Antônio Percope de ANDRADE
}

Departamento do Aparelho Locomotor, Faculdade de Medicina da Universidade Federal de Minas Gerais-UFMG, 30130-100 Belo Horizonte-MG, Brasil

\begin{abstract}
Resumo
Introdução: A doença articular degenerativa, osteoartrose, é, no geral, a principal causa de deficiência física entre idosos. A dor e a limitação funcional provocadas por essa condição, especialmente nos membros inferiores, apresentam forte correlação com a redução na qualidade de vida dessas pessoas. A artroplastia total do joelho é um procedimento terapêutico de excelentes resultados na artrose do joelho, cada vez mais prevalente. Objetivo: Conhecer a qualidade de vida antes e depois desse procedimento sob a perspectiva do paciente. Material e método: Estudo qualitativo, descritivo, realizado no Hospital das Clínicas da Universidade Federal de Minas Gerais, Belo Horizonte, com 50 pacientes submetidos a artroplastia total do joelho. A tabulação dos dados ocorreu por meio da utilização de três figuras metodológicas: ideia central, expressões chave e o discurso sujeito coletivo. Resultados: Melhora importante da dor, ganho funcional, maior sensação de segurança e menor dependência de medicamentos com função analgésica foram vantagens destacadas. Ainda que uma parcela mantenha queixas álgicas em outros segmentos após o procedimento, a satisfação com a prótese foi significativa entre os pacientes, com descrições originais sobre a qualidade de vida nos dois momentos exemplificando essa constatação. Conclusão: A satisfação com a artroplastia total do joelho é significativa entre os pacientes em vários domínios, claramente visível em uma exploração de caráter qualitativo.
\end{abstract}

Descritores: Avaliação da Pesquisa em Saúde; Osteoartrite; Artroplastia do Joelho; Qualidade de Vida.

\section{Abstract}

Introduction: Degenerative joint disease, osteoarthritis, is, in general, the main cause of physical disability among the elderly. The pain and functional limitation caused by this condition, especially in the lower limbs, present a strong correlation with a reduction in their quality of life. Total knee arthroplasty is a therapeutic procedure with excellent results in knee arthritis, which is increasingly prevalent. Objective: The objective was to assess the quality of life before and after this procedure from the patient's perspective.

Methods: A qualitative, descriptive study was performed at Hospital das Clínicas, Federal University of Minas Gerais, Belo Horizonte, Brazil, with 50 patients who underwent total knee arthroplasty. The tabulation of data occurred through the use of three methodological figures: central idea, key expressions and collective subject discourse. Results: Significant improvement of pain, functional gain, greater sensation of safety and less dependence on drugs with analgesic function were the main benefits. Although a portion maintains painful complaints in other segments after the procedure, the satisfaction with the prosthesis was significant among the patients, with original descriptions about the quality of life in the two moments, exemplifying this finding. Conclusion: Satisfaction with total knee arthroplasty is significant among patients in several domains, clearly visible in a qualitative exploration.

Descriptors: Health Research Evaluation; Osteoarthritis; Arthroplasty, Replacement, Knee; Quality of Life.

\section{Resumen}

Introducción: La enfermedad articular degenerativa, la osteoartritis, es, en general, la principal causa de deficiencia física entre ancianos. El dolor y la limitación funcional provocada por esta condición, especialmente en los miembros inferiores, presentan una fuerte correlación con la reducción en la calidad de vida de esas personas. La artroplastia total de la rodilla es un procedimiento terapéutico de excelentes resultados en la artrosis de la rodilla, cada vez más prevalente. Objetivo: Se objetivó conocer la calidad de vida antes y después de ese procedimiento desde la perspectiva del paciente. Material y método: Estudio cualitativo, descriptivo, realizado en el Hospital de las Clínicas de la Universidad Federal de Minas Gerais, Belo Horizonte, con 50 pacientes sometidos a artroplastia total de la rodilla. La tabulación de los datos ocurrió por medio de la utilización de tres figuras metodológicas: idea central, expresiones clave y el discurso del sujeto colectivo. Resultados: Mejora importante del dolor, aumento funcional, mayor sensación de seguridad y menor dependencia de medicamentos con función analgésica fueron ventajas destacadas. Aunque una parcela mantiene quejas álgicas en otros segmentos después del procedimiento, la satisfacción con la prótesis fue significativa entre los pacientes, con descripciones originales sobre la calidad de vida en los dos momentos ejemplificando esa constatación. Conclusión: La satisfacción con la artroplastia total de la rodilla es significativa entre los pacientes en varios dominios, claramente visible en una exploración de carácter cualitativo.

Descriptores: Evaluación de la Investigación en Salud; Osteoartritis; Artroplastia de Reemplazo de Rodilla; Calidad de Vida.

\section{INTRODUÇÃO}

Artroplastia é um procedimento cirúrgico para restaurar o movimento indolor de uma articulação e restabelecer a função dos músculos, dos ligamentos e das outras estruturas de tecidos moles que controlam essa região anatômica ${ }^{1}$. A cirurgia de substituição articular total é indicada em casos de indivíduos que possuem articulação com artrose dolorosa, incapacitante e refratária ao tratamento conservador $^{1}$. Estudo epidemiológico conduzido por Lenza et al. ${ }^{2}$ aponta que a maioria dos pacientes
$(81,3 \%)$ submetidos a artroplastia total do joelho (ATJ) tem 65 anos ou mais. O principal diagnóstico pré-operatório que motivou a cirurgia foi a osteoartrose $(84,9 \%)$. Dentre as comorbidades, a doença clínica mais frequentemente diagnosticada no pré-operatório foi hipertensão arterial sistêmica $(50,4 \%)^{2}$. Kurtz et al. ${ }^{3}$ projetam que a demanda por artroplastia primária do joelho terá um aumento de $673 \%$, de 450.000 em 2005 para 3,48 milhões em 2030, nos Estados Unidos da America ${ }^{2}$. 
O conceito de qualidade de vida $(\mathrm{QV})$ teve sua base relacionada à aquisição de bens materiais e à capacidade das populações em adquiri-los para um bem-estar social. Com o desenvolvimento das sociedades e do sistema capitalista, esse termo passou a ser ampliado devido à necessidade de avaliar o contexto mais universalizado do bem estar físico, mental e social. Segundo a Organização Mundial de Saúde, o conceito de QV mais utilizado é "a percepção individual de um completo bem-estar físico, mental e social" ${ }^{4,5}$.

A doença articular degenerativa, osteoartrose, é, no geral, a principal causa de deficiência física entre idosos. A dor e a limitação funcional provocadas por essa condição, especialmente nos membros inferiores, apresentam forte correlação com a redução na $\mathrm{QV}$ dessas pessoas ${ }^{6,7}$.

Os métodos atuais de avaliação dos resultados da ATJ são baseados principalmente em sinais e sintomas clínicos, no exame físico e na avaliação radiográfica. Esse tipo de avaliação não contempla todos os aspectos do tratamento, já que não é capaz de detectar as reais necessidades e expectativas do paciente, tais como mudanças na $\mathrm{QV}$, nas relações sociais e no seu meio ambiente ${ }^{6,8-11}$.

Durante a última década houve aumento no interesse em avaliar a maneira pela qual os pacientes percebem o impacto da doença, isto é, a qualidade de vida relacionada à saúde. $\mathrm{A} \mathrm{QV}$ relacionada à saúde é, portanto, uma construção multidimensional que captura o impacto do estado de saúde, incluindo doença e tratamento em três domínios: físico, psicológico e função social ${ }^{12}$.

O objetivo desse trabalho foi conhecer quais as perspectivas dos pacientes em relação a qualidade de vida apresentada antes e após o tratamento com a prótese total do joelho, de uma maneira qualitativa, centrada no paciente.

\section{MATERIAL E MÉTODO}

Optou-se pela pesquisa qualitativa do tipo exploratória, adotando como metodologia a Teoria das Representações Sociais (TRS). Esta tem grande aplicabilidade na área de saúde, apreendendo os aspectos mais subjetivos que permeiam os problemas dessa área. Para conhecer e descrever as considerações dos pacientes sobre a qualidade de vida antes e depois da artroplastia total do joelho, sob o referencial das TRS, o Discurso do Sujeito Coletivo (DSC) constituiu-se como método escolhido.

A pesquisa qualitativa, segundo Minayo ${ }^{13}$, responde às questões particulares, considerando como sujeito de estudo pessoas pertencentes a um grupo e com uma determinada condição social, com um universo de significados, valores, crenças e atitudes. A pesquisa exploratória é realizada em áreas nas quais há pouco conhecimento acumulado e sistematizado, constituindo-se na primeira etapa de uma investigação mais ampla, sendo desenvolvida quando o tema é pouco explorado. Por sua natureza de sondagem, não comporta hipóteses que, todavia, podem surgir durante ou ao final da pesquisa ${ }^{13}$.

Realizou-se então a entrevista individual com duas questões semiestruturadas, após assinatura do termo de consentimento livre e esclarecido (TCLE), obedecendo-se à resolução n. ${ }^{\circ} 466 / 12$ do Conselho Nacional de Saúde, que trata de pesquisa envolvendo seres humanos, sendo que as questões éticas deste trabalho foram orientadas pelas suas diretrizes e levadas, pelo investigador, ao conhecimento dos sujeitos da pesquisa. A obtenção do TCLE é um processo que tem por objetivo permitir que o sujeito da pesquisa compreenda os procedimentos, riscos, desconfortos, benefícios e direitos envolvidos, dando condições para que haja uma decisão autônoma.

O estudo, realizado no período de 01 de abril de 2017 a 01 de novembro de 2017, teve como sujeitos 50 pacientes submetidos à ATJ, acompanhados no ambulatório de joelho do Hospital das Clínicas da Universidade Federal de Minas Gerais. As entrevistas investigaram quais as considerações dos pacientes sobre a qualidade de vida antes e depois da ATJ. Cada entrevista foi gravada em fita e depois transcrita para análise. Para a análise e apresentação dos resultados utilizou-se o DSC, redigido na primeira pessoa do singular, composto por expressões chaves $(\mathrm{ECH})$ que tiveram as mesmas ideias centrais (IC) e mesma ancoragem (AC), obedecendo-se rigorosamente a ordem das seguintes etapas:

- $1^{\mathrm{a}}$ etapa: análise das respostas e posterior transcrição das mesmas;

- $2^{\text {a }}$ etapa: leitura individual das respostas de cada entrevistado, seguida da leitura separada de todas as respostas para a questão analisada;

- $3^{\text {a }}$ etapa: transcrição das respostas para a questão 1 , sendo indicadas as IC, que representaram a descrição das ECH e não a sua interpretação. Mesmo procedimento para todas as questões;

- $4^{\text {a }}$ etapa: transcrição individual de cada ideia central com as suas respectivas ECH;

- $5^{\text {a }}$ etapa: extração do tema de cada uma das perguntas, agrupando-se a suas respectivas IC. Finalmente, construção dos DSC separadamente de cada ideia central, com as suas respectivas ECH.

\section{RESULTADOS}

Quanto à caracterização da amostra por gênero, obtivemos 40 pacientes do sexo feminino e 10 do sexo masculino. Quanto à lateralidade, 21 próteses à direita, 14 a esquerda e 15 bilaterais. Quanto ao tempo transcorrido desde a cirurgia, 23 casos haviam sido operados há menos de 1 ano e 27 há mais de 1 ano. Quanto ao tratamento prévio realizado, 41 pacientes relataram fisioterapia, 6 pacientes relataram infiltração, 31 pacientes o uso de 
de anti-inflamatório e 6 acupuntura.

Quando abordados com a primeira pergunta (como era sua qualidade de vida antes da prótese?) a ideia central referida por $74 \%$ dos entrevistados foi dor, sendo que $56 \%$ referiram limitação funcional, $22 \%$ citaram insegurança, $20 \%$ mencionaram dependência de medicações, $6 \%$ discorreram sobre deformidade apresentada no membro; 1 paciente $(2 \%$ da amostra) disse que a qualidade de vida antes da cirurgia era melhor. As ideias centrais são mostradas no Gráfico 1.

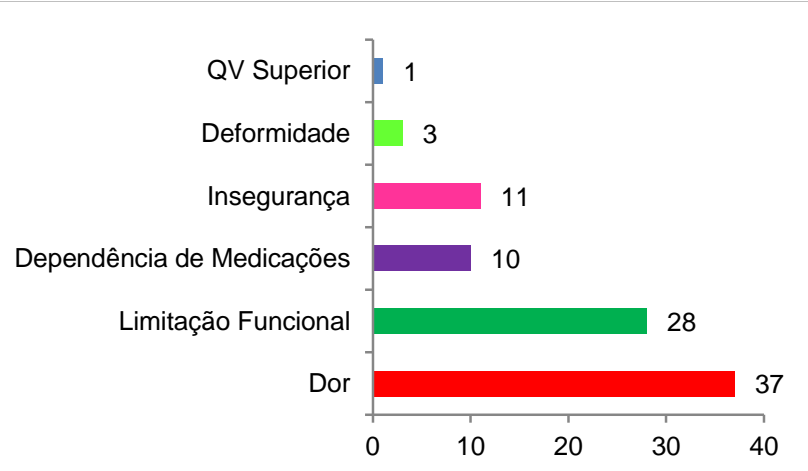

Gráfico 1: Qualidade de vida antes da prótese: ideias centrais para a primeira abordagem.

As ideias centrais (IC), palavras-chave e discurso do sujeito coletivo para a primeira abordagem encontram-se listados abaixo:

o IC: Dor - $74 \%$

DSC: "Minha qualidade de vida era péssima, de tanta dor. Os joelhos doíam muito, por isso, era muito ruim(...) Doía muito. A dor era insuportável aqui no osso. Ficava doendo quentinho, como se tivesse uma febre nele. Doía muito para subir e descer escadas(...) Doía muito para movimentar. Sentia muita dor na perna. Eu chorava de dor o tempo todo(...) Esse joelho doía tanto, que as vezes tinha vontade de morrer. Era 24 horas doendo(...) A dor era direto. Andava, estava doendo; parada, estava doendo; deitada, estava doendo; sentada, doía. Eu gritava de dor. Eu vivia no médico, de tanto que doía(...) Fazia as coisas em casa com dor. Minha vida era chorar de tanta dor. A dor era nota dez. Dor para todo lado. Doía dia e noite(...)"

- IC: Dependência de Medicações - $20 \%$

DSC: "Eu usava muito remédio para dor. Tomava morfina direto, minha mão enchia de remédios para dormir(...) Os remédios que eu tomava já não faziam efeito. Era remédio demais. Tomava remédio para dor, mas não cortava não, só aliviava(...) Eu vivia a base de remédio. Não existia remédio que curasse essa dor(...)"

○ IC: Limitação Funcional - 56\%

DSC: "Não conseguia fazer nada, só ficava na cama. Para ajoelhar era muito difícil. Quase não saia de casa porque não conseguia caminhar. Para andar, tinha quase que $\operatorname{arrastar}(. .$.$) No trabalho, me$ incomodava demais. Tive que usar bengala. Não conseguia encontrar maneira adequada de dormir(...) Não dava para trabalhar em pé, tinha que sentar o tempo todo. Na minha casa já não conseguia fazer nada direito. Ás vezes não conseguia por o pé no chão. Tinha muitas coisas que eu não conseguia fazer(...) Eu tinha que andar só em superfícies planas, e mesmo assim não andava com desenvoltura. $\mathrm{O}$ joelho me atrapalhava o tempo todo no trabalho. Conseguia fazer as coisas com muita dificuldade: varrer a casa era só escorada em uma cadeira. Cheguei a precisar de cadeira de rodas(...) Ficava mais era deitada. As pernas eram muito pesadas; parecia que pesavam uns cem quilos(...)"

○ IC: Deformidade - 6\%

DSC: "Era terrível, um joelho estava quase encontrando o outro. Eu entortei, as pernas estavam abrindo(...) Minha perna era muito torta."

○ IC: Insegurança - 22\%

DSC: "Quando andava, parecia que eu estava pisando longe do chão; estava usando bengala, porque tinha medo de cair. Sentia que minhas pernas não estavam equilibrando(...) Se eu andesse um pouco mais rápido, eu caia. Era bem complicado ficar de pé. Escorava na bengala; se a bengala escorregasse, eu caia(...) Preferia andar em superfícies planas. Caia no chão sem parar. Tinha muito medo de cair e quebrar a perna(...) Tomei um tombo, e ai tive que usar bengalas. Sentia como se as pernas fossem falhar, que eu fosse cair, como se elas não estivessem segurando(...)"

○ IC: Era Melhor - 2\%

DSC: "Ótima, minha vida era tranquila. Antes, era bem melhor, com certeza! Mesmo com o joelho arrebentado era bem melhor do que com a prótese. Jogava até bola, dirigia, trabalhava normal. Agora não mais."

$\mathrm{Na}$ segunda abordagem (como é a sua qualidade de vida após a prótese?), $56 \%$ dos entrevistados referiram como ideia central a melhora da dor, $36 \%$ a melhora da função, $18 \%$ enfatizaram sobre a dor em outro segmento, $8 \%$ disseram não ter tido tempo suficiente para avaliar e 1 paciente (2\%) referiu piora da qualidade de vida. As ideias centrais são mostradas no Gráfico 2.

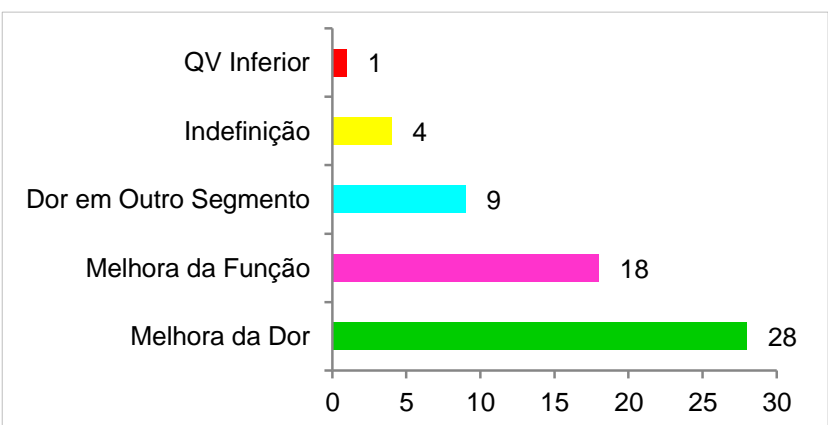

Gráfico 2: Qualidade de vida após a prótese: ideias centrais para a segunda abordagem. 
As ideias centrais (IC), palavras-chave e discurso do sujeito coletivo (DSC) para a segunda abordagem encontram-se listados abaixo:

○ IC: Melhora da Dor - $56 \%$

DSC: "Estou me sentindo muito bem. Não sinto mais nenhuma dor no joelho. Valeu a pena. A sensação é muito melhor(...) A dor melhorou mil por cento. Não doi nada. Em vista da dor que eu sentia, melhorou muito(...) Uma benção a dor sumir assim. Não tomo mais nenhum analgésico. Depois da cirurgia doeu muito, mas agora com a fisioterapia, melhorou bem(...)"

○ IC: Melhora da Função - 36\%

DSC: "Antes não fazia nada em casa, agora faço a maioria das coisas. Custava a ajoelhar para as orações, agora está bem melhor. Já dobro o joelho muito melhor(...) Eu subo, desço escada,varro,limpo a casa. A sensação é muito melhor. Não me atrapalha mais para andar e fazer as coisas do meu dia a dia(...) Estou andando de bicicleta, trabalho, melhorou muito. Agora lavo vasilha, lavo roupa, cozinho. Estou dançando até forró(...) Conseguir voltar a dirigir é o que mais me deixou feliz. Já estou me mexendo muito melhor. Larguei a bengala, não preciso mais de apoio(...) Eu não conseguia limpar debaixo de uma cama, agora consigo. Consigo varrer um terreiro. Fazer esse dia a dia, antes não fazia, hoje consigo (...)"

○ IC: Tempo Insuficiente para Avaliar - 8\%

DSC: "Ainda está no começo, mas vai indo. Vamos ver se vai melhorar(...) Está muito precoce, ainda não notei nada. Ainda não estou fazendo nada porque é muito recente(...)Não posso falar que está melhor ou pior porque está muito recente. Por isso ainda preciso de remédios. Seis meses ainda só,né. Por isso não estou forçando muito ele ainda(...)"

○ IC: Ênfase na Dor de Outros Segmentos - 18\%

DSC: "Agora é a outra perna que doi(...) Agora o que interfere mais é a coluna. Ando escorada(...) O pé está doendo muito agora. Engraçado, melhora uma coisa, piora a outra(...) Agora o que me incomoda é a outra perna. O problema é que ainda preciso da muleta por causa do outro joelho(...) Essa outra perna agora está atrapalhando muito minha recuperação. E a coluna também sente, né(...) Acho que a única coisa que está atrapalhando minha recuperação mais rápida é o fêmur. O fêmur atrapalha bastante(...)"

- IC: Preferia Antes - 2\%

DSC: "Agora doi demais. Dia e noite. Não consigo dormir. Preferia antes da cirurgia. Antes doía nota 10 , hoje a dor é nota 12 . Não tenho ânimo para mais nada."

\section{DISCUSSÃO}

A abordagem das condições de vida do paciente antes da prótese envolve necessariamente a observância de que a QV se refere à percepção que cada pessoa tem de sua posição na vida, dentro do contexto de cultura e sistema de valores nos quais ela vive e em relação a suas metas, expectativas e padrões sociais. Nesse contexto, as condições de saúde impactam diretamente na percepção das pessoas sobre a sua qualidade de vida.

Cassetari ${ }^{14}$ avaliou a qualidade de vida de idosos portadores de osteoartrose do joelho e observou que a queixa de limitações funcionais foi um achado significativo. Os resultados obtidos pelo autor também apontaram limitações em várias atividades como: levantar da cadeira, ficar em pé, curvar-se ao chão e sentar-se. No presente trabalho, $56 \%$ dos entrevistados também relataram limitação funcional como ideia central ao ser indagado sobre a qualidade de vida previamente à prótese. Relatos como "para ajoelhar era muito difícil" exemplificam essa colocação.

A dor advinda da artrose resulta de interações complexas entre o dano articular, inflamação e anormalidades no processamento da dor. Revisão sistemática conduzida por Woolf $^{15}$ em 2011 considerou a presença de hipersensibilidade em pacientes portadores de osteoartrose do quadril, joelho e primeira articulação carpo-metacárpica. Embora os analgésicos prescritos para controle da dor atuem em diferentes mecanismos, podem falhar em prover adequado alívio da dor ou podem até mesmo serem descontinuados devido a eventos adversos ${ }^{16}$. Em nosso estudo, a dor foi a principal ideia central gerada sobre a qualidade de vida antes da prótese, referida por $74 \%$ dos entrevistados.

Medicamentos como os anti-inflamatórios não hormonais são modalidades frequentemente utilizadas no tratamento da artrose do joelho. Dados da literatura sobre a utilização de antiinflamatórios para artrose não são conclusivos quanto às diferenças nos tipos e dosagens dos mesmos, ressaltando que a seleção desses fármacos deve ser baseada na indicação do médico e no custo financeiro ${ }^{17}$. Apesar de serem as medicações mais acessíveis aos pacientes, os efeitos colaterais gastrointestinais, cardiovasculares e renais devem ser relembrados. Nesse estudo, 20\% dos pacientes referiram a dependência de medicações para alívio de dor antes da realização da prótese de joelho.

A osteoartrose, com o passar dos anos, afeta o aparato capsuloligamentar da articulação, permitindo a ocorrência de desvios angulares e deformidades. É típico encontrar pacientes que referem instabilidade subjetiva uma vez que há alongamento do ligamento cruzado anterior e dos colaterais ${ }^{18-20}$. Tal achado é compatível com o estudo em questão, em que $22 \%$ dos pacientes referiram sensação de insegurança e $6 \%$ relataram a presença 
de deformidades quando indagados sobre a qualidade de vida previamente a prótese.

Ao se contextualizar a qualidade de vida do paciente após a instalação da prótese é preciso considerar que previsões apontam que a proporção de pessoas com mais de 60 anos deverá dobrar até 2050 . $\mathrm{O}$ aumento da quantidade de pessoas com artrose vai aumentar a demanda por ATJs, o que irá configurar um sério problema de saúde pública ${ }^{21}$.

Os resultados técnicos de uma ATJ são excelentes. A sobrevida do paciente é de $99 \%$ após 1 ano e $84 \%$ em 10 anos $^{22}$. Todavia, indicações tradicionais de sucesso cirúrgico como o implante usado podem não refletir a perspectiva do paciente em relação à cirurgia.

Estudos mostraram melhora considerável da função de pacientes submetidos à ATJ, o que refletiu em benefícios para as atividades cotidianas ${ }^{23-25}$. Apontam que entre $37 \%$ e $56 \%$ dos operados permanecem muito ativos e capazes de desempenhar atividades físicas como serviços de casa e práticas esportivas $^{26,27}$, além de melhorar a habilidade para caminhar. Dados da literatura destacam que até $71 \%$ dos pacientes tinham dificuldade para caminhar previamente e $76 \%$ foram capazes de caminhar sem padrões anômalos de marcha após a cirurgia ${ }^{28,29}$. Em nosso estudo, corroborando com esses resultados, $36 \%$ dos pacientes referiram melhora funcional como ideia central ao serem indagados sobre a qualidade de vida após a prótese total do joelho.

Satisfação com os resultados da ATJ também foi observada em outros estudos com $75 \%$ dos pacientes satisfeitos com o resultado da ATJ em 11,7 $\operatorname{anos}^{28-30}$. Consequentemente, $79,5 \%$ dos pacientes indicaram que se submeteriam novamente à cirurgia $^{27}$. Em nosso estudo, a principal ideia central encontrada para 56\% dos entrevistados sobre a qualidade de vida após a cirurgia foi satisfação com a melhora da dor.

Embora a maioria dos estudos demonstre melhora da dor, da função e da qualidade de vida, até $20 \%$ dos pacientes que se submetem a prótese total do joelho permanecem insatisfeitos. Dor pós-operatória persistente presente, por mais de 6 meses, é frequentemente a principal causa de insatisfação $^{31}$. Os resultados obtidos nessa pesquisa mostram que um paciente $(2 \%)$ referiu melhor qualidade de vida antes de ser submetido ao procedimento. O trecho "agora doi demais. Não consigo dormir direito" atesta a piora da dor e o decréscimo da função e qualidade de vida do paciente. Enquanto a maioria dos pacientes experimenta melhora da dor 3 meses após o procedimento, aproximadamente $20 \%$ persistem com dor a longo prazo de acordo com Beswick et al ${ }^{32}$.

A predisposição à dor crônica é multifatorial e inclui além da severidade da dor pós-operatória, o fator de risco mais importante, dor pré-existente e fatores psicológicos ${ }^{33}$. A dor pré-operatória, seja no sítio operatório, seja em outro segmento, é um fator de risco conhecido ${ }^{34}$. $18 \%$ dos pacientes entrevistados em nosso estudo, quando indagados sobre a qualidade de vida após a artroplastia, enfatizaram a existência de dor em outro segmento corpóreo, como a coluna, pés, joelho contralateral e quadril.

\section{CONCLUSÃO}

Foi notória a mudança de qualidade de vida dos pacientes submetidos a artroplastia total do joelho. Melhora importante da dor, ganho funcional, maior sensação de segurança e menor dependência de medicamentos com função analgésica foram destacados. Ainda que uma parcela mantenha queixas álgicas em outros segmentos após o procedimento, a satisfação com a prótese foi significativa entre os pacientes, com descrições originais sobre a qualidade de vida nos dois momentos exemplificando essa constatação.

\section{REFERÊNCIAS}

1. Canale T. Cirurgia Ortopédica de Campbell. 10. ed. Barueri: Manole; 2006.

2. Kurtz S, Ong K, Lau E, Mowat F, Halpern M. Projections of primary and revision hip and knee arthroplasty in the United States from 2005 to 2030. J Bone Joint Surg Am. 2007; 89(4):780-85.

3. Lenza M, Ferraz Sde B, Viola DC, Garcia Filho RJ, CendorogloNeto M, Ferretti M Epidemiology of total hip and knee replacement: a cross-sectional study. Einstein. 2013; 11(2):197-202.

4. Seidi EMF, Zannon CMLC. Qualidade de Vida e Saúde: aspectos conceituais e metodológicos. Cad Saúde Pública; 2004; 20(2):580-8.

5. Oliveira MP, Ferreira AMA, Cordeiro RXR, Lima JA. Quality of life and social characteristics of patients submitted to total knee arthroplasty. Rev bras ortop. 2015; 47(1):77-82.

6. Silva RR, Santos AAM, Carvalho Júnior JS, Matos MA. Quality of life after total knee arthroplasty: systematic review. Rev Bras Ortop. 2014; 49(5):520-27.

7. Torres TM, Ciconelli RM. Epidemiologia da osteoartrose. In: Pardini AG, Souza JMG, editors. Clínica ortopédica - atualizacão em osteoartroses. Rio Janeiro: Guanabara Koogan; 2005.

8. Noble PC, Conditt MA, Cook KF, Mathis KB. The John Insall Award: Patient expectations affect satisfaction with total knee arthroplasty. Clin Orthop Relat Res. 2006; 452:35-43.

9. Bayley KB, London MR, Grunkemeier GL, Lansky DJ. Measuring the success of treatment 
in patient terms. Med Care. 1995; 33(4 suppl):AS226-35.

10. Bullens PH, van Loon CJ, de Waal Malefijt MC, Laan RF, Veth RP. Patient satisfaction after total knee arthroplasty: a comparison between subjective and objective outcome assessments. J Arthroplasty. 2001; 16(6):740-47.

11. Hudak PL, McKeever P, Wright JG. Understanding the meaning of satisfaction with treatment outcome. Med Care. 2004; 42(8):718-25.

12. Leidy NK, Revicki DA, Genesté B. Recommendations for evaluating the validity of quality of life claims for labeling and promotion. Value Health. 1999; 2(2):113-27

13. Minayo MCS (org). Pesquisa social: teoria, método e criatividade. Petrópolis: Vozes; 1994.

14. Cassetari MR. Osteoartrose em joelhos como fator limitante para a qualidade de vida em idosos [dissertação]. Botucatu: Universidade Estadual Paulista (UNESP), Faculdade de Medicina de Botucatu; 2008.

15. Woolf CJ. Central sensitization: implications for the diagnosis and treatment of pain. Pain. 2011; 152(3 Suppl):S2-15.

16. Schnitzer TJ. Update on guidelines for the treatment of chronicusculoskeletal pain. Clin Rheumatol. 2006; 25(Suppl 1):S22-9.

17. Watson M, Brookes ST, Kirwan J, Faulkner A. Non-aspirin, non-steroidal anti-inflammatory drugs for treating osteoarthritis of the knee. Cochrane Database Syst Rev. 2000; (2):CD000142.

18. Duivenvoorden T, Brouwer RW, Baan A, Bos PK, Reijman M, Bierma-Zeinstra SM et al: Comparison of closing-wedge and openingwedgehigh tibial osteotomy for medial com-partment osteoarthritis ofthe knee: a randomized controlled trial with a six-year follow-up. J Bone Joint Surg Am. 2014; 96(17): 1425-32.

19. Pogliacomi F, Defilippo M, Guardoli A, Scaravella E: High tibial osteotomy:our experience with hemicallotasis method. Acta Biomed. 2014; 85( Suppl 2):85-90.

20. Stuart M, Backstein D, Logan M, Muellner T. Osteotomy about the Knee: International roundtable discussion. Scott WN. Insall \& Scott Surgery of the Knee. 5 th ed. Philadelphia: Elsevier; 2012.p.944-51.

21. United Nations Department of Economic and Social Affairs. World population prospects: the 2012 revision. New York: 2011

22. Cross M, Lapsley H, Barcenilla A, Parker D, Coolican M, March L. Patient expectationsof hip and knee joint replacement surgery and postoperative health status. Patient. 2009;
2(1):51-60.

23. Joshi AB, Gill G. Total knee arthroplasty in nonagenarians. J Arthroplasty. 2002; 17(6):681-4.

24. Bourne RB, McCalden RW, MacDonald SJ, Mokete L, Guerin J. Influence ofpatient factors on TKA outcomes at 5 to 11 years followup. Clin Orthop Relat Res. 2007; 464:27-31.

25. Seng C, Yeo SJ, Wee JL, Subanesh S, Chong $\mathrm{HC}$, Lo NN. Improved clinicaloutcomes after high-flexion total knee arthroplasty: a 5-year follow-up study. J Arthroplasty. 2011; 26(7):1025-30.

26. Wright RJ, Sledge CB, Poss R, Ewald FC, Walsh ME, Lingard EA. Patientreportedoutcome and survivorship after Kinemax total knee arthroplasty. J Bone Joint Surg Am. 2004; 86-A(11):2464-70.

27. Nuñez M, Lozano L, Nuñez E, Segur JM, Sastre $S$, Maculé $F$ et al. Total knee replacement and health-related quality of life: factors influencing long term outcomes. Arthritis Rheum. 2009; 61(8):1062-69.

28. Pagnano MW, McLamb LA, Trousdale RT. Total knee arthroplasty for patients 90 years of age and older.Clin Orthop Relat Res. 2004; 418:179-83.

29. Wright RJ, Sledge CB, Poss R, Ewald FC, Walsh ME, Lingard EA. Patientreportedoutcome and survivorship after Kinemax total knee arthroplasty. J Bone Joint Surg Am. 2004; 86(11):2464-70.

30. Nuñez M, Nuñez E, del Val JL, Ortega R, Segur JM, Hernández MV et al. Health-related quality of life in patients with osteoarthritis aftertotal knee replacement: factors influencing outcomes at 36 months of follow-up. Osteoarthritis Cartilage. 2007; 15(9):1001-7.

31. Carli F, Charlebois P, Stein B, Feldman L, Zavorsky G, Kim DJ et al. Randomized clinical trial of prehabilitationin colorectal surgery. $\mathrm{Br} \mathbf{J}$ Surg. 2010; 97(8):1187-97.

32. Beswick AD, Wylde V, Gooberman-Hill R, Blom A, Dieppe P. What proportion of patients report long-term pain after total hip or knee replacement for osteoarthritis? A systematic review of prospective studies in unselected patients. BMJ Open. 2012; 2(1):e000435.

33. Kehlet H, Jensen TS, Woolf CJ. Persistent postsurgical pain: risk factors and prevention. Lancet 367(9522):1618-25.

34. Liu SS, Buvanendran A, Rathmell JP, Sawhney $\mathrm{M}$, Bae JJ, Moric $\mathrm{M}$ et al. A cross-sectional survey on prevalence andrisk factors for persistent postsurgical pain 1 year after total hipand knee replacement. Reg Anesth Pain Med. 2012; 37(4):415-22. 
CONFLITO DE INTERESSES

Os autores declaram não haver conflitos de interesse.

AUTOR PARA CORRESPONDENCIA

Eli Ávila Souza Júnior

elijr42@yahoo.com.br

Submetido em 09/05/2018

Aceito em 06/06/2018 